\title{
Two-Dimensional FDTD Analysis of a Pulsed Microwave Confocal System for Breast Cancer Detection: Fixed-Focus and Antenna-Array Sensors
}

\author{
Susan C. Hagness, * Member, IEEE, Allen Taflove, Fellow, IEEE, and Jack E. Bridges, Life Fellow, IEEE
}

\begin{abstract}
A novel focused active microwave system is investigated for detecting tumors in the breast. In contrast to $\mathrm{X}$-ray and ultrasound modalities, the method reviewed here exploits the breast-tissue physical properties unique to the microwave spectrum, namely, the translucent nature of normal breast tissues and the high dielectric contrast between malignant tumors and surrounding lesion-free normal breast tissues. The system uses a pulsed confocal technique and time-gating to enhance the detection of tumors while suppressing the effects of tissue heterogeneity and absorption. Using published data for the dielectric properties of normal breast tissues and malignant tumors, we have conducted a two-dimensional (2-D) finite-difference timedomain (FDTD) computational electromagnetics analysis of the system. The FDTD simulations showed that tumors as small as $2 \mathrm{~mm}$ in diameter could be robustly detected in the presence of the background clutter generated by the heterogeneity of the surrounding normal tissue. Lateral spatial resolution of the tumor location was found to be about $0.5 \mathrm{~cm}$.
\end{abstract}

Index Terms - Biomedical electromagnetic imaging, cancer, FDTD methods, microwave antenna arrays, numerical analysis, scattering, tumors.

\section{INTRODUCTION}

$\mathbf{T}$ WHERE IS considerable recent debate as to whether or not women under 50 years of age should have X-ray mammograms. This debate arises from the need to detect breast cancer in its earliest stage. Early detection leads to longest survival and greatest patient comfort. While mammography is recognized as the preferred method to detect breast cancer, it fails to detect as many as $20 \%$ of the malignant tumors. Further, it may be uncomfortable or threatening to many of the patients, especially with the public perception that repeated Xray mammograms increase the risk of cancer. Other modalities such as ultrasound and magnetic resonance imaging (MRI) are either less effective or too costly. Based in part on our work reported in this paper, we believe that pulsed confocal microwave technology can complement mammography by remedying most of the above noted deficiencies.

Manuscript received November 4, 1997; revised May 27, 1998. This work was supported by the National Institutes of Health (NIH) under Phase-I SBIR Grant 1R43CA67598 01A2. Asterisk indicates corresponding author.

*S. C. Hagness is with the Department of Electrical and Computer Engineering, University of Wisconsin-Madison, 1415 Engineering Drive, Madison, WI 53706 USA (e-mail: hagness@engr.wisc.edu).

A. Taflove is with the Department of Electrical and Computer Engineering, McCormick School of Engineering and Applied Science, Northwestern University, Evanston, IL 60208 USA.

J. E. Bridges is with Interstitial, Inc., Park Ridge, IL 60068 USA.

Publisher Item Identifier S 0018-9294(98)08848-X.

\section{A. Physical Basis of the Method}

The confocal microwave breast cancer detection technology considered here [1] is based upon two fundamental properties of breast tissues at microwave frequencies, as reported in the literature (discussed in detail in Section II).

Property 1: Microwaves interact with biological tissues primarily according to the tissue water content. This is a different interaction mechanism than for X-rays. The relevant physicalproperties contrast between malignant tumors and normal breast tissues is significantly greater for microwaves than for either X-rays or ultrasound, approaching an order of magnitude. This large dielectric contrast causes malignant tumors to have significantly greater microwave scattering cross sections than normal tissues of comparable geometry.

Property 2: Microwave attenuation in normal breast tissue is less than $4 \mathrm{~dB} / \mathrm{cm}$ up to $10 \mathrm{GHz}$. This may permit existing microwave equipment having standard sensitivity and dynamic range to detect tumors located up to about $5 \mathrm{~cm}$ beneath the skin. The microwave attenuation and phase characteristic of normal breast tissue is such that constructive addition is possible for wide-bandwidth backscattered returns using broadaperture confocal-imaging techniques. The confocal technique suppresses returns from spurious scatterers such as a vein interposed between the tumor and the surface of the breast.

\section{B. Technology Basis}

Confocal microscopy has long been used in optics [2]. We are investigating a novel adaptation for tumor detection that: 1) focuses an illuminating microwave signal at a potential tumor site; and 2) efficiently collects the microwave energy scattered by the tumor by refocusing it at the point of origin of the illumination. This provides spatial resolution of both transmitted and received signals.

The confocal microwave technology is based on previous work under contract to the United States Federal Aviation Administration [3] and [4]. That work resulted in the successful design and testing of a confocal $94-\mathrm{GHz}$ FM chirp radar to detect and image metal and plastic weapons concealed under passengers' clothing. Redesign of the chirp-radar system at 4-10 $\mathrm{GHz}$ permits propagation of a focused, pulsed, lowpower beam into the human breast. Upon encountering a tumor, the microwave energy is backscattered because the tumor has significantly greater dielectric permittivity and conductivity than normal breast tissue. This energy is efficiently 
collected by the sensor antenna which lies at the out-ofbreast focal point. By systematically scanning the in-breast focal point to lie within a set of preselected voxels, a threedimensional (3-D) image of the tumor can be constructed.

Here, the lateral spatial resolution is dependent upon the size of the physical or synthetic aperture of the sensor antenna. The depth resolution is dependent upon the use of time-gating ("range-gating"). Range-gating can be achieved either directly by using a narrow impulsive illumination or indirectly by using FM-chirp-radar pulse synthesis. The sharpness of the lateral and depth resolutions, and the consequent suppression of clutter caused by the normally occurring permittivity variations in healthy breast tissue, is a function of the complexity of the microwave equipment/signal-processing software and the medical application.

\section{Complementary Nature Relative to X-Ray Mammography}

The confocal microwave technology under consideration contrasts tissues primarily according to their water content, and utilizes the backscattered return from the entire tumor. This may complement X-ray mammography when used to sense early-stage or other types of malignancies that might have appreciable water content but few microcalcifications detectable by $\mathrm{X}$ rays.

The confocal microwave technology would cause zero ionizing radiation exposure. It could be relatively comfortable since it would require access to only one side of the breast. These safety and comfort features might facilitate the use of this technology for both frequent screening of the public and frequent monitoring of the progress of the treatment protocol for an individual patient.

\section{Background Literature}

Nonmammographic breast-imaging techniques other than ultrasound and MRI have been studied, and several reviews of such alternative technologies are available [5]-[7]. Although extensively studied, passive microwave radiometry (thermography) [8] was not considered promising in two of these reviews [5], [6].

The heterogeneous nature of the breast presents formidable technical barriers in applying active, nonradiometric microwave techniques to tumor detection. With the exception of the comprehensive review, Medical Applications of Microwave Imaging [9], there is sparse published literature in this area. More recently, there is published work reporting biomedical imaging approaches based on unfocused microwave backscatter [10], [11] and matrix-inversion of multistatic, multisensor impedance data [12], or microwave data [13].

The specific use of a short-pulse, wide bandpass, confocal microwave system employing backscattering to detect or image breast cancers appears not to have been investigated previously. However, some of the basic principles related to this technology have been investigated for other purposes. Examples include the work of Young and Peters [14], who demonstrated a video-pulse ground-penetrating radar to identify buried gas pipes and land mines. To form a 3-D image,

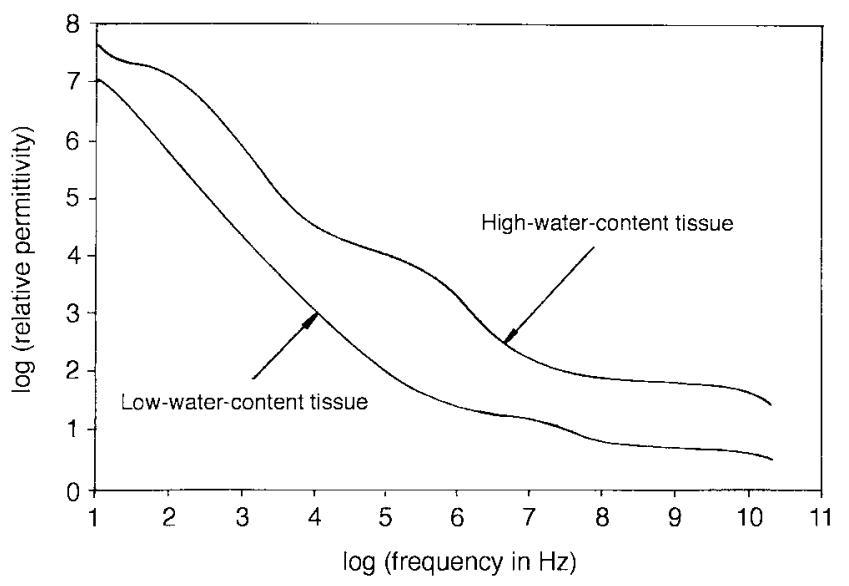

Fig. 1. Comparison of the permittivity of high-water-content tissue such as muscle with low-water-content tissue such as fat as a function of frequency according to Gabriel et al. [23]-[26].

they moved a sensor along the earth's surface, recorded the time history of the backscattered pulse at each sensor location, and used the method of time-shifting the received pulse to develop data for imaging the underground target. Cribbs [15] worked on the same problem, applying an FM synthetic pulse technique similar to that used in Bridges' work [3], [4]. The recent (July, 1997) North American Radio Science Meeting in Montreal, Canada, sponsored a session on impulse radar. Of the eleven papers, six concerned aspects of ground-penetrating radar to locate land mines or similar buried targets [16]-[21].

The signal processing proposed for the confocal microwave technology (tested via numerical modeling later in this paper) employs a robust approach analogous to that used in videopulse ground-penetrating radar [14] and geophysical seismic prospecting [22], wherein impulsive returns from subsurface features observed at a set of receiver locations along the surface are time-shifted to add coherently. The time-shifted data are then processed to provide a 3-D image of the subsurface scatterers.

\section{Breast Tissue Dielectric Properties}

\section{A. Dielectric Contrast Between Malignant Tumors and Surrounding Normal Breast Tissue}

Measurements of 30 different tissue types by Gabriel et al. [23]-[26] indicate that the relative dielectric permittivity, $\epsilon_{r}$, and conductivity, $\sigma$, of high-water-content tissues (such as muscle or malignant tumors) are about an order of magnitude greater than those of low-water-content tissues (such as fat or normal breast tissue). As illustrated in Figs. 1 and 2, this contrast between high- and low-water-content tissues persists over the entire radio-frequency (RF) spectrum from power frequencies through millimeter waves.

Joines et al. [27] measured to $0.9 \mathrm{GHz}$ the $\epsilon_{r}$ and $\sigma$ of freshly excised tissues from the colon, kidney, liver, lung, breast, and muscle. Each tissue sample was taken from four to seven different patients, and each sample was measured at three different positions. Chaudhary et al. [28] measured to $3 \mathrm{GHz}$ the $\epsilon_{r}$ and $\sigma$ of normal and malignant breast tissues 


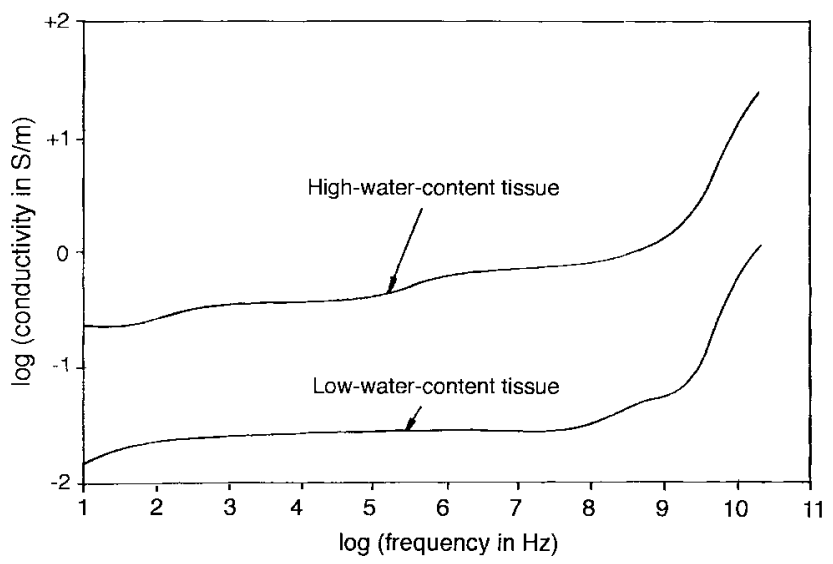

Fig. 2. Comparison of the conductivity of high-water-content tissue such as muscle with low-water-content tissue such as fat as a function of frequency according to Gabriel et al. [23]-[26].

obtained from 15 patients. The data of Joines et al. at 0.9 $\mathrm{GHz}$ indicate that $\sigma$ of malignant breast tumors exceeds that of normal breast tissue by $6.4: 1$, and $\epsilon_{r}$ of malignant breast tumors exceeds that of normal breast tissue by $3.8: 1$. Joines et al. further found that for breast tissues of the same type, the dielectric contrast between malignant and normal tissues is greatest for the mammary gland. The data of Chaudhary et al. up to $3 \mathrm{GHz}$ indicate corresponding malignant tumorto-normal breast tissue ratios of $4.7: 1$ and $5: 1$, in good agreement with Joines et al.

\section{B. Malignant Tumor Properties}

This paper addresses the issue of detecting malignant tumors, especially those with little or no microcalcifications. Some benign tumors may also have a high water content and could produce a backscatter response similar to that generated by malignant tumors. However, characterizing and analyzing such benign tumors is an extensive subject by itself and is not considered in this paper. Here, we focus only on malignant tumor properties.

Foster and Schepps [29], Rogers et al. [30], and Pelso et al. [31] separately measured $\epsilon_{r}$ and $\sigma$ of malignant tumors and found values above $1 \mathrm{GHz}$ that are almost the same as for normal high-water-content tissues such as muscle. In some cases, $\epsilon_{r}$ and $\sigma$ for malignant tumors were significantly greater than for normal muscle tissues, especially at frequencies below $1 \mathrm{GHz}[30]$.

Swarup et al. [32] studied the onset of the high values of $\epsilon_{r}$ and $\sigma$ in malignant tumors by measuring MCA1 fibrosarcoma mouse tumors at 7, 15, and 30 days after inception. No significant variation of $\epsilon_{r}$ and $\sigma$ was seen with tumor age. While the larger tumors exhibited a necrotic interior, they showed little difference in $\epsilon_{r}$ and $\sigma$ above $0.5 \mathrm{GHz}$.

Surowiec et al. [33] performed measurements of cm-size malignant human breast tumors and adjacent tissues and found an increase in $\epsilon_{r}$ and $\sigma$ of the normal breast tissue near malignant tumors. This effect may be caused by infiltration or vascularization. It could enlarge the microwave scattering cross-section and thereby aid in the confocal microwave detection of the tumor.

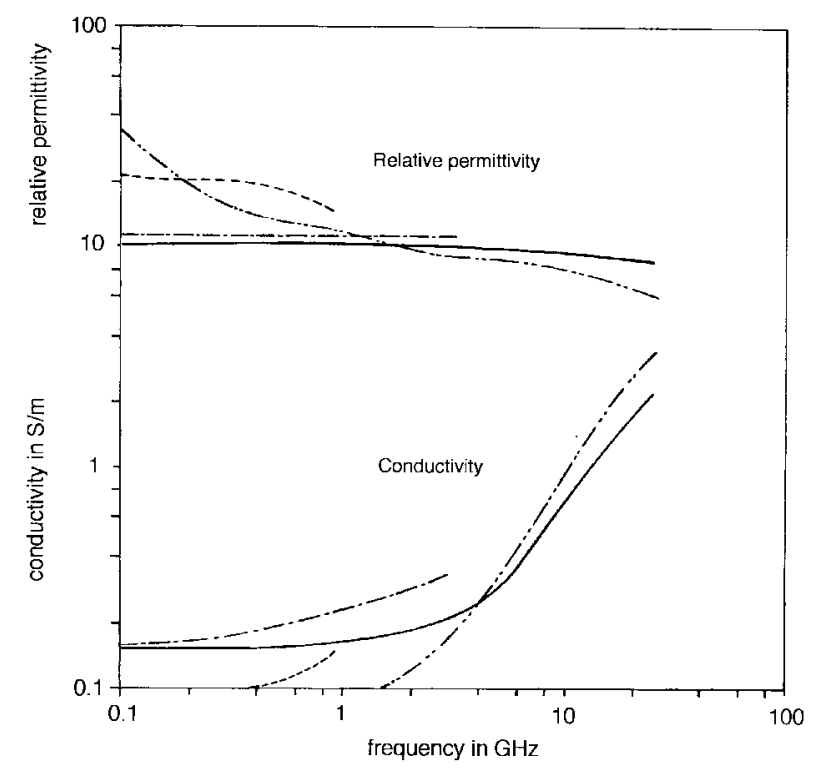

Fig. 3. Relative permittivity and conductivity as a function of frequency for normal breast tissue as measured by Joines et al. [27] (dashes) and by Chaudhary et al. [28] (dash-dot) compared with Foster's and Schwan's Debye model (solid) and empirical model (dash-dot-dot) [29].

\section{Normal Breast Tissue Dielectric Properties}

Fig. 3 graphs measured data for $\epsilon_{r}$ and $\sigma$ as a function of frequency for normal breast tissue as measured by Joines $e t$ al. [27] to $1 \mathrm{GHz}$ and by Chaudhary et al. [28] to $3 \mathrm{GHz} .{ }^{1}$ Both groups' data are in good agreement, apparently because both used protocols that preserved the tissue water content and carefully segregated malignant from normal tissues.

The measured data of Joines et al. and Chaudhary et al. were extrapolated to higher frequencies using the Debye model as given in Foster and Schwan [34]

$$
\epsilon_{r}(f)=\epsilon_{\infty}-\frac{j \sigma}{2 \pi f \epsilon_{0}}+\frac{\epsilon_{s}-\epsilon_{\infty}}{1+j\left(f / f_{p}\right)}
$$

Here, $f$ is the frequency in $\mathrm{Hz}, \epsilon_{0}=8.854 \times 10^{-12} \mathrm{~F} / \mathrm{m}$, and the parameters $\epsilon_{s}=10, \epsilon_{\infty}=7, f_{p}=2.5 \times 10^{10} \mathrm{~Hz}$, and $\sigma=0.15 \mathrm{~S} / \mathrm{m}$ were chosen to well match the measured data of Joines et al. and Chaudhary et al. Equation (1) yields the solid curves shown in Fig. 3 extending to $30 \mathrm{GHz}$.

We used the empirical relationship between the microwave dielectric parameters and the moisture content developed by Foster and Schwan to further confirm the Debye fit. For an approximate $10 \%$ moisture content, their empirical model is as follows:

$$
\begin{aligned}
\epsilon_{r}^{\prime} & =1.71 f^{1.13}+\frac{\epsilon_{s}-4}{1+(f / 25)^{2}} \\
\sigma & =1.35 \sigma_{0.1} f^{0.13}+0.00222 f^{2}\left[\frac{\epsilon_{s}-4}{1+(f / 25)^{2}}\right] .
\end{aligned}
$$

Here, $f$ is the frequency in $\mathrm{GHz}$ and the parameters $\sigma_{0.1}=$ 0.05 and $\epsilon_{s}=8.5$ were chosen from the Foster and Schwan data. Equations (2) and (3) and the selected parameters yielded

\footnotetext{
${ }^{1}$ The $\sigma$ data in Fig. 3 were normalized from $25^{\circ} \mathrm{C}$ as measured to $37^{\circ} \mathrm{C}$ according to the procedure noted in [34].
} 


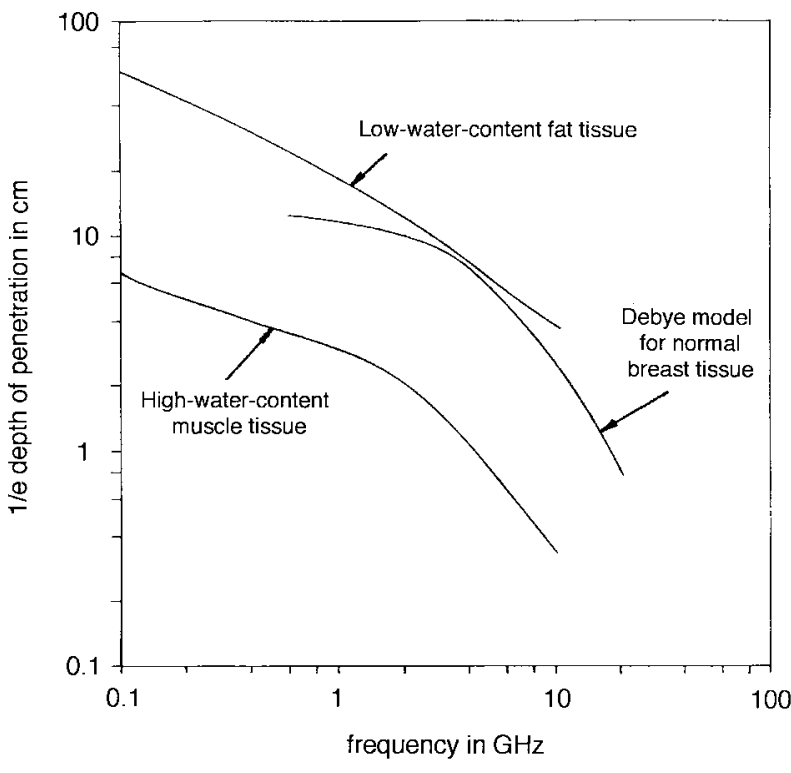

Fig. 4. Depth of penetration as a function of frequency as given by Johnson and Guy for low-water-content fat tissue and for high-water-content muscle tissue compared to the depth of penetration for normal breast tissue developed from the Debye model of Fig. 3.

the dashed-double-dotted curves shown in Fig. 3 extending to $30 \mathrm{GHz}$.

We note from Fig. 3 generally good agreement between the measured data and the Debye model for both $\epsilon_{r}$ and $\sigma$ above $0.3 \mathrm{GHz}$. The empirical model provides a somewhat better fit for $\epsilon_{r}$ than does the Debye model but does not track well the measured $\sigma$. However, above $4 \mathrm{GHz}$, the empirical model yields values that are consistent with those developed from the Debye model.

These curve-fit procedures allow a reasonably accurate extrapolation of the measured dielectric data for normal breast tissues up to $10 \mathrm{GHz}$ and a subsequent calculation of $\delta$, the $1 / e(-8.686 \mathrm{~dB})$ depth of penetration. At $6 \mathrm{GHz}$, the Debye model yields values of $\epsilon_{r} \cong 9$ and $\sigma \cong 0.4 \mathrm{~S} / \mathrm{m}$. These values were used in the nondispersive computational electromagnetics models discussed later. Malignant tumors were assumed to have values of $\epsilon_{r}=50$ and $\sigma=7 \mathrm{~S} / \mathrm{m}$ at this frequency, similar to those of muscle. Fig. 4 graphs $\delta$ as a function of frequency as published by Johnson and Guy [35] for lowwater-content fat tissue and for high-water-content muscle tissue. Also plotted is $\delta$ for normal breast tissue developed from the Debye model data of Fig. 3. The Debye data for normal breast tissue yields penetration depths that agree well with the values in [35] for low-water-content fat tissue. From these data, normal breast tissue is seen to exhibit a penetration depth of $2.5-3 \mathrm{~cm}$ at $10 \mathrm{GHz}$, equivalent to a microwave path loss of about $3.5 \mathrm{~dB} / \mathrm{cm}$.

\section{Heterogeneity of Normal Breast Tissue}

Joines et al. [27] noted the standard error on the mean for the aggregate of all their measurements as follows: $\pm 5 \%$ for $\epsilon_{r}$ of normal tissues and $\pm 7 \%$ for $\epsilon_{r}$ of malignant tissues; $\pm 7 \%$ for $\sigma$ of normal tissues and $\pm 9 \%$ for $\sigma$ of malignant tissues. The results of Chaudhary et al. [28] exhibited a similar spread over the average values by no more than $\pm 8 \%$. These narrow data ranges occurred despite the large $0.5-\mathrm{cm}-1.0-\mathrm{cm}$ tissue-sample dimensions which caused each group's samples to contain an unknown mixture of fat, mammary gland, and duct tissues. The key implication here is that normal breast tissue dielectric heterogeneity at an observable distance scale of about $0.5 \mathrm{~cm}$ is bounded in a small fractional range about the median value. Accordingly, for the computational electromagnetics models discussed later, we used a $\pm 10 \%$ variability range about the median $\epsilon_{r}$ and $\sigma$ for a given tissue type at this distance scale, this being an approximate upper bound to the data variability measured by both Joines et al. and Chaudhary et al. We note that specific dielectric values for mammary duct and gland tissues are not available. For purposes of our modeling, the values for the ducts and glands were assumed to be $15 \%$ higher than those of normal breast tissue, that is, somewhat beyond the upper limit of the measured variability range.

\section{E. Skin and Veins}

Gabriel et al. found that, for either wet or dry skin, $30<$ $\epsilon_{r}<40$ and $1<\sigma<10 \mathrm{~S} / \mathrm{m}$ from $1-10 \mathrm{GHz}$. While some dielectric property data exist for blood, none were found for vein walls. For our computational models, we assumed that the dielectric properties of a vein are the same as those of muscle.

\section{F. Breast Geometry}

The depth of a typical normal, nonlactating human breast is on the order of $5 \mathrm{~cm} \mathrm{[36]-[38].} \mathrm{This} \mathrm{suggests} \mathrm{that} \mathrm{a} \mathrm{mildly-}$ compressed breast would span less than $5 \mathrm{~cm}$ between the skin surface and the rib cage. Further, almost $50 \%$ of all breast tumors occur in the quadrant near the armpit where the breast is less than about 2.5-cm deep [39]. Accordingly, we have based our computational models of the confocal microwave system on detecting tumors to depths of about $5 \mathrm{~cm}$ with a typical depth of 3-4 cm.

\section{MODELING OF THE FIXED-FocUS ELLIPTICAL REFLECTOR SYSTEM}

As the first step in the detailed systems analysis, a fixedfocus confocal microwave system employing a metal elliptical reflector was computationally modeled in two dimensions using the finite-difference time-domain (FDTD) solution of Maxwell's equations [40], [41] for the transverse magnetic case. The reflector was specified with one focal point at a monopole antenna element and one in a breast half-space 3.8 $\mathrm{cm}$ below the surface. (In such two-dimensional (2-D) models, all material structures in the computational space, including the antenna, are assumed to be infinitely long. Thus, there is no ground return for the antenna and it is termed a "monopole.")

Fig. 5(a) illustrates the FDTD model of this system. This model used a uniform grid with square unit cells as fine as $0.2 \mathrm{~mm}$ in the highest-resolution simulations. The reflector was assumed filled with lossless dielectric $\left(\epsilon_{r}=9, \sigma=0\right)$ matching the nominal breast permittivity, and was located at the surface of a half-space of normal breast tissue $\left(\epsilon_{r}=9, \sigma=\right.$ $0.4 \mathrm{~S} / \mathrm{m})$. No skin layer was modeled. The monopole antenna was excited by a $270-$ ps Gaussian pulse multiplying a $6-\mathrm{GHz}$ 


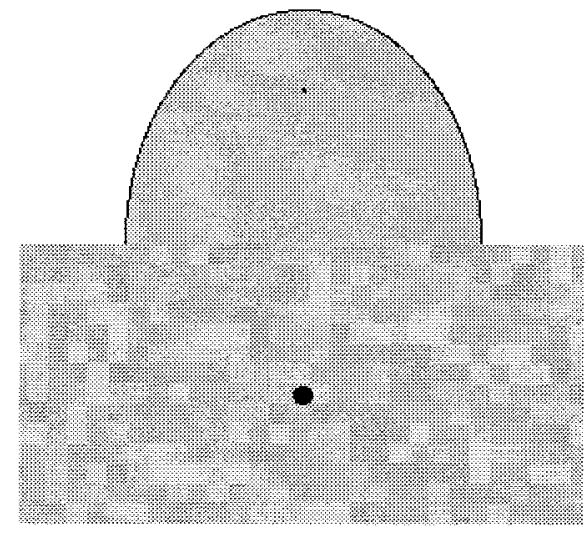

(a)

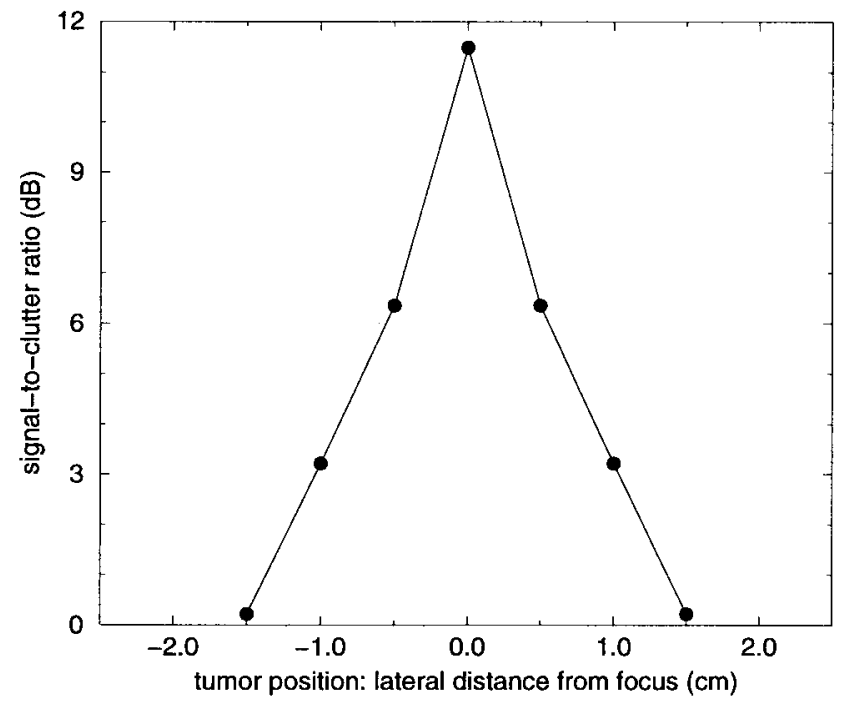

(c)

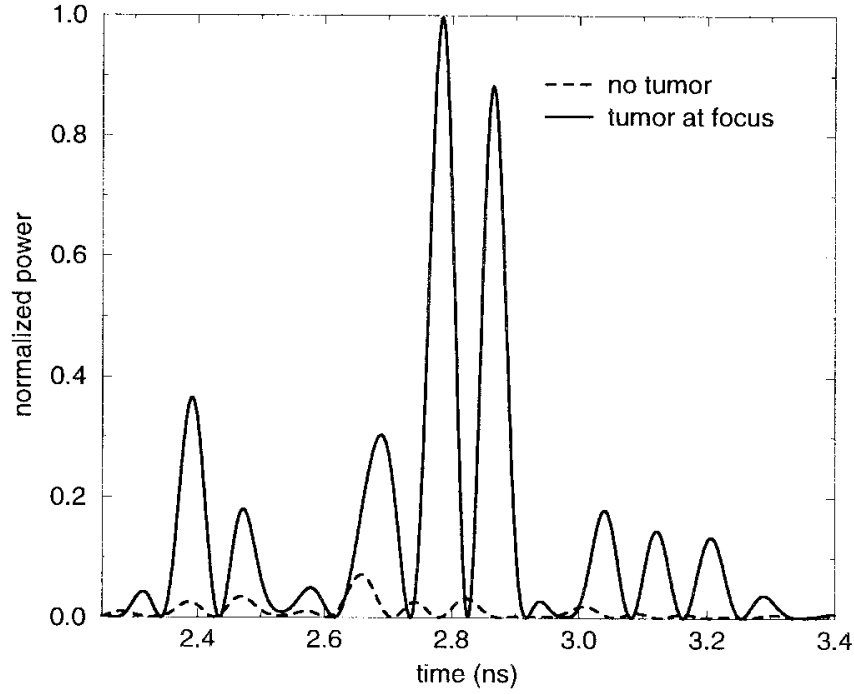

(b)

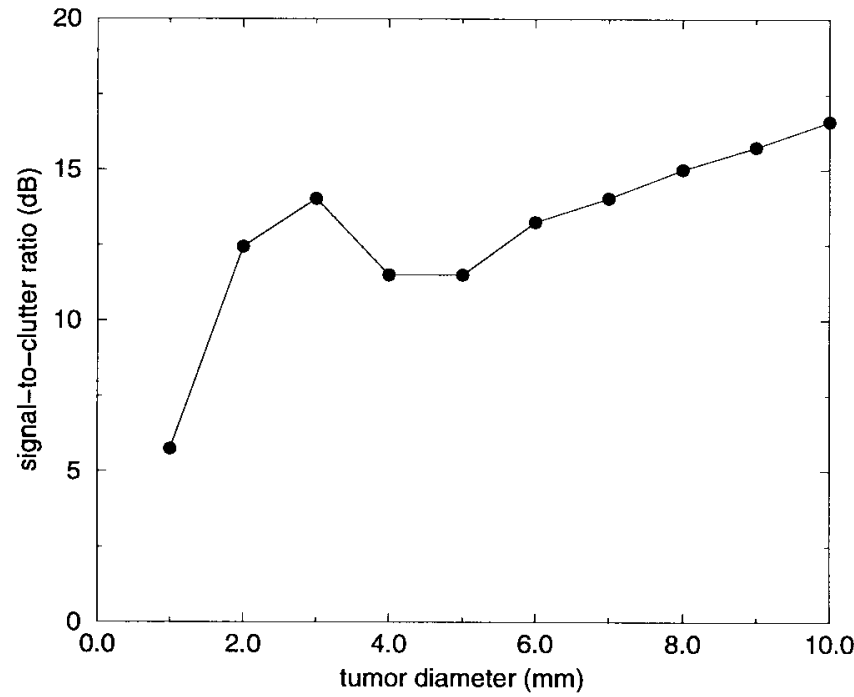

(d)

Fig. 5. (a) 2-D FDTD computational model of the elliptical reflector system showing the heterogeneous breast tissue model ( $\pm 10 \%$ variations) and a $0.5-\mathrm{cm}$-diameter tumor located at the in-breast focus $3.8 \mathrm{~cm}$ beneath the surface, (b) FDTD-computed time-domain waveforms of the backscattered response with and without the $0.5-\mathrm{cm}$-diameter tumor present at the in-breast focus, (c) signal-to-clutter (S/C) ratio for the backscattered response of the 0.5 -cm-diameter tumor as a function of the tumor's lateral distance from the in-breast focus, and (d) S/C ratio for the backscattered response of a tumor at the in-breast focus as a function of the tumor's size.

sinusoid that passed through zero at the peak of the Gaussian. This signal has zero dc content and a Gaussian, doublesideband (DSB) suppressed-carrier spectrum symmetric about $6 \mathrm{GHz}$. The full-width spectral bandwidth at half-maximum extends from 4 to $8 \mathrm{GHz}$. A circular tumor $\left(\epsilon_{r}=50, \sigma=7\right.$ $\mathrm{S} / \mathrm{m})$ was assumed located at the in-breast focus.

\section{A. $\pm 10 \%$ Random Heterogeneity of the Normal Breast Tissue}

To simulate the heterogeneity of the normal breast tissue as measured by Joines et al. [27] and Chaudhary et al. [28], $\pm 10 \%$ random fluctuations of $\epsilon_{r}$ and $\sigma$ were assigned to the breast tissue half-space in a checkerboard pattern. Specifically, as shown in Fig. 5(a), each square block of grid cells spanning $5 \times 5 \mathrm{~mm}$ was randomly assigned a value of $\epsilon_{r}$ and a value of $\sigma$ in a $\pm 10 \%$ range centered about the nominal. This resulted in random, peak $\pm 20 \%$ jump discontinuities of the normalbreast-tissue $\epsilon_{r}$ and $\sigma$ at the scale of the tumor diameter. FDTD modeling was performed for a) no tumor present, to establish the background clutter; b) variable location of a tumor having a $0.5-\mathrm{cm}$ fixed diameter; and c) variable diameter of a tumor having a fixed location at the in-breast focal point. The signal-to-clutter (S/C) ratio was obtained by comparing the peak backscattered responses of the heterogeneous breast model with and without the presence of the tumor.

Fig. 5(b) depicts the calculated time waveforms of the backscattered power response for this model with and without the $0.5-\mathrm{cm}$-diameter tumor present at the in-breast focus 3.8 $\mathrm{cm}$ below the surface. Upon forming the ratio of the peak backscattered pulse amplitude with the tumor present to the peak backscattered pulse amplitude without the tumor present, 


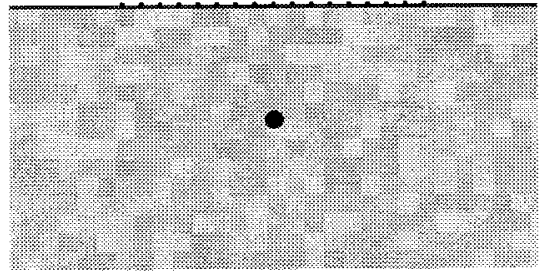

(a)

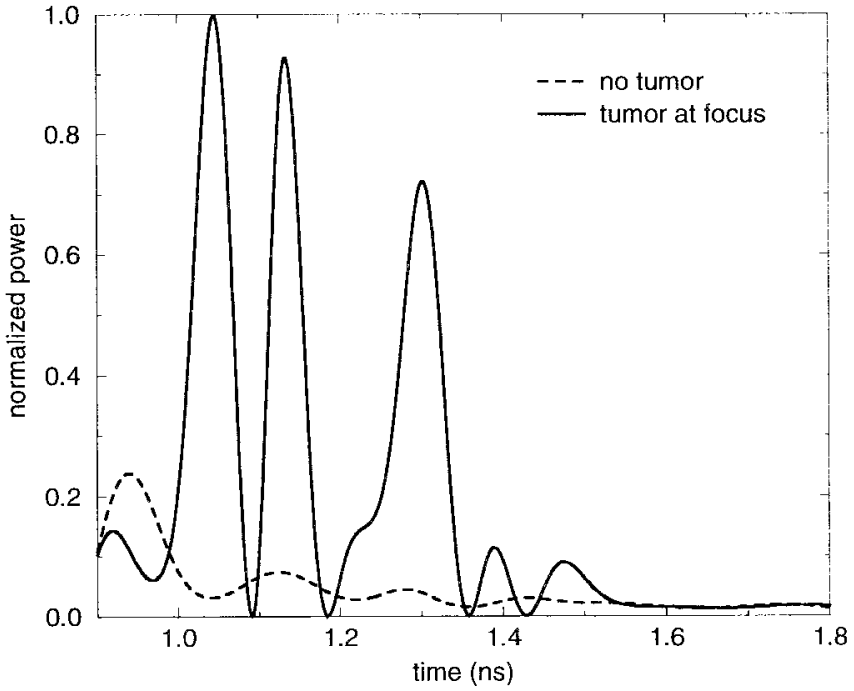

(b)

Fig. 6. (a) 2-D FDTD computational model of the 17-position coherent-addition antenna array showing a 1.0-mm-thick layer of skin, the heterogeneous breast tissue model $( \pm 10 \%$ variations), and a $0.5-\mathrm{cm}$-diameter tumor located at the synthetic focus $3.0 \mathrm{~cm}$ beneath the surface and (b) FDTD-computed time-domain waveforms resulting from time-shifting and summing the backscattered responses.

the $\mathrm{S} / \mathrm{C}$ ratio is found to be $12 \mathrm{~dB}$. Fig. 5(c) graphs the calculated $\mathrm{S} / \mathrm{C}$ for the $0.5-\mathrm{cm}$ diameter tumor as a function of the tumor's lateral position from the focus for a constant depth of $3.8 \mathrm{~cm}$. We infer from this figure that the lateral resolution in locating the tumor in the presence of the clutter is about $0.5 \mathrm{~cm}$. Fig. 5(d) graphs the calculated $\mathrm{S} / \mathrm{C}$ for a tumor fixed in position at the in-breast focus as a function of the tumor's size. We infer from this figure that tumors having diameters as small as $0.2 \mathrm{~cm}$ can yield responses that are 12 $\mathrm{dB}$ above the background clutter due to the random $\pm 10 \%$ tissue heterogeneity.

\section{B. $\pm 20 \%$ Random Heterogeneity of the Normal Breast Tissue}

We repeated the above study for an increased heterogeneity of the normal breast tissue of $\pm 20 \%$ about the nominal, more than twice that experimentally observed by Joines et al. [27] and Chaudhary et al. [28]. This resulted in random, peak $\pm 40 \%$ jump discontinuities of the normal-breast-tissue $\epsilon_{r}$ and $\sigma$ at the scale of the tumor diameter. While the computed $\mathrm{S} / \mathrm{C}$ ratios were reduced by $5-6 \mathrm{~dB}$ relative to the $\pm 10 \%$ heterogeneity case, the $\mathrm{S} / \mathrm{C}$ remained greater than $6 \mathrm{~dB}$ for tumor diameters of $0.2 \mathrm{~cm}$ or larger. Further, the system's lateral resolution was unchanged (about $0.5 \mathrm{~cm}$ ).

\section{MODELING OF THE COHERENT-ADDITION ANTENNA ARRAY}

The next step in the FDTD simulations involved replacing the elliptical reflector antenna with a 17-element electronically scanned monopole array spanning $8 \mathrm{~cm}$, as shown in Fig. 6(a). The monopoles were assumed to be spaced at $0.5-\mathrm{cm}$ intervals along the surface of a 0.1 -cm-thick skin layer having $\epsilon_{r}=36$ and $\sigma=4 \mathrm{~S} / \mathrm{m}$. A $0.5-\mathrm{cm}$-diameter circular tumor was assumed to be located $3.0 \mathrm{~cm}$ directly below the center of the monopole array surrounded by normal breast tissue having the random checkerboard heterogeneity of Fig. 5(a).
The modeling procedure began by simulating an impulsive excitation of each monopole element, one at a time, using the same 4-GHz bandwidth DSB waveform assumed in Section III. Next, the backscattered pulse response of the tumor observed at that element was calculated and stored. Last, the 17 individual backscatter waveforms were timeshifted to achieve a coherent sum of the tumor's backscattered response for the desired focal point. We note that varying the distribution of these time shifts across the array scans the focal point. Time shifting and summing are seen to be postprocessing operations performed on the previously calculated FDTD backscatter data records.

\section{A. $\pm 10 \%$ Random Heterogeneity of the Normal Breast Tissue}

Fig. 6(b) shows the received power waveform upon coherently summing the responses of the 17 monopoles. A S/C ratio in the same $10-\mathrm{dB}$ range previously calculated for the elliptical reflector is seen. This implies that the clutter suppression of the fixed-focus elliptical reflector can be achieved by a variablefocus monopole array implementing time-shifting and coherent addition. In additional modeling work, we studied the effect of the skin conductivity varying over the range $0.5-5 \mathrm{~S} / \mathrm{m}$. The $\mathrm{S} / \mathrm{C}$ ratio was found to vary only to second order (within about $\pm 1 \mathrm{~dB}$ ), implying robustness of the time-shifting and coherent-addition process relative to skin conductivity.

\section{B. Effect of a Directly Interposed Vein}

Fig. 7(a) illustrates the geometry of interposing a $0.2-\mathrm{cm}-$ diameter vein $\left(\epsilon_{r}=50, \sigma=7 \mathrm{~S} / \mathrm{m}\right)$ directly between the $0.5-\mathrm{cm}$-diameter tumor and the sensor array at a depth of $1.5 \mathrm{~cm}$. Fig. 7(b) graphs the received power waveform upon coherently summing over the 17 monopoles. It was found that range-gating permits distinguishing the vein response from that of the tumor. The $\mathrm{S} / \mathrm{C}$ ratio of the tumor response is not degraded. 


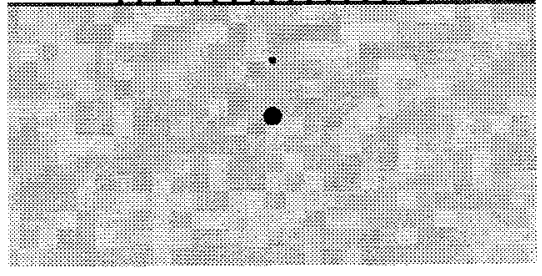

(a)

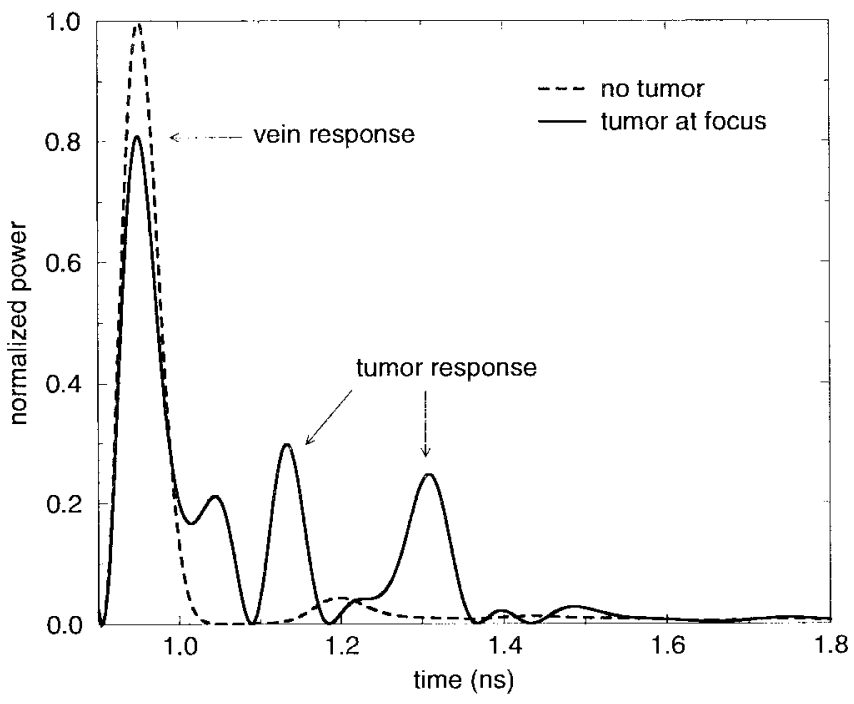

(b)

Fig. 7. (a) 2-D FDTD computational model of the 17-position coherent-addition antenna array shown in Fig. 6(a), but with a 0.2-cm-diameter vein interposed between the skin and the tumor and (b) FDTD-computed time-domain waveforms resulting from time-shifting and summing the backscattered responses.

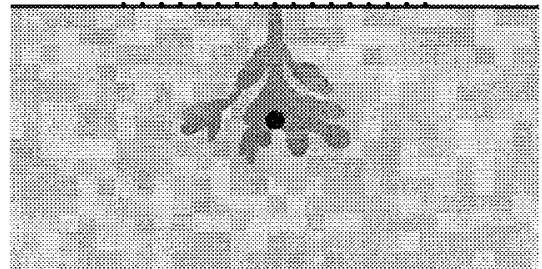

(a)

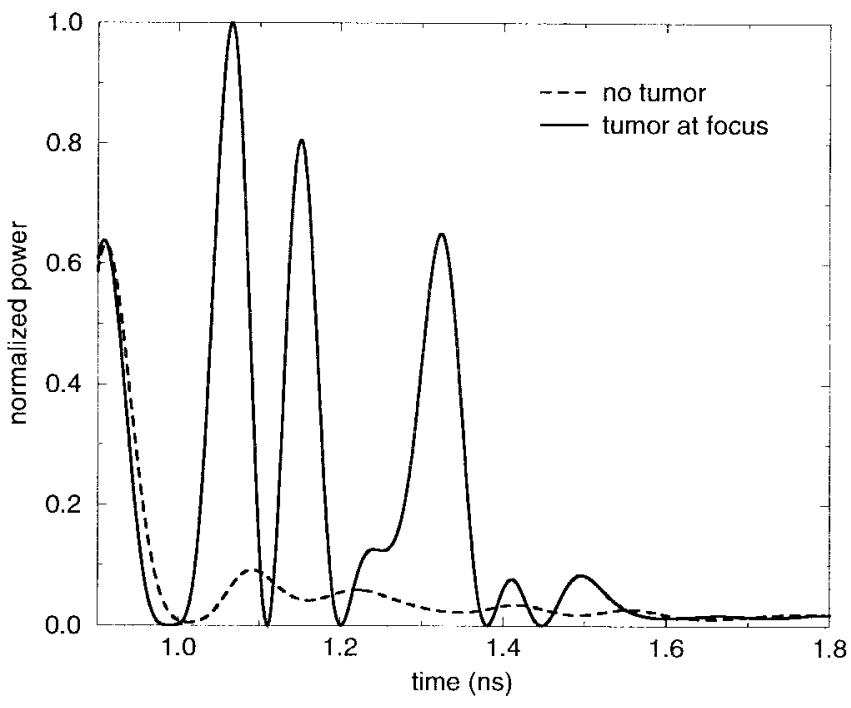

(b)

Fig. 8. (a) 2-D FDTD computational model of the 17-position coherent-addition antenna array shown in Fig. 6(a), but with mammary glands and ducts interposed between the skin and the tumor and (b) FDTD-computed time-domain waveforms resulting from time-shifting and summing the backscattered responses.

\section{Effect of a Directly Interposed Gland Cluster}

Fig. 8(a) illustrates the geometry of interposing a cluster of mammary glands and ducts directly between the $0.5-\mathrm{cm}-$ diameter tumor and the sensor array. Here, a simulated gland cluster was modeled in the FDTD grid by positioning a group of small ellipses having $15 \%$ higher $\epsilon_{r}$ and $\sigma$ than normal breast tissue. Fig. 8(b) graphs the received power waveform upon coherently summing over the 17 monopoles. In the time period after $1.0 \mathrm{~ns}$, the FDTD-calculated S/C ratio is seen to be unaltered from the 10-dB level obtained before, with no gland cluster present. Apparently, the modeled gland cluster generated a backscattered return that propagated incoherently to the multiple observation locations. Similar to the background $\pm 10 \%$ random heterogeneity in the checkerboard pattern, this return was rejected upon coherent addition in favor of the tumor's signature.

Fig. 9(a)-(d) provide a vivid indication of the cluttersuppression action of the coherent-addition process. Here are shown four samples of the FDTD-computed raw backscattered data (before time-shifting and summing) that were used to generate the composite power waveform of Fig. 8(b). We see that no single backscattered waveform gives an indication of an embedded tumor response. However, simple time-shifting of the waveforms according to the nominal propagation delay between each monopole position and the desired focal point in an assumed uncluttered tissue medium suffices to extract the tumor response upon arithmetic summation. 


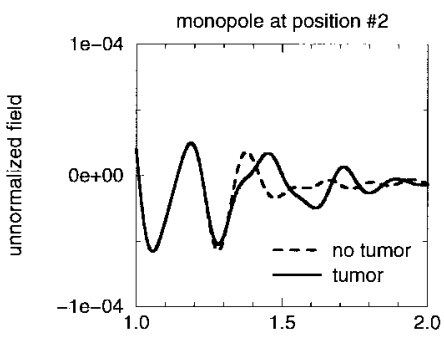

(a)

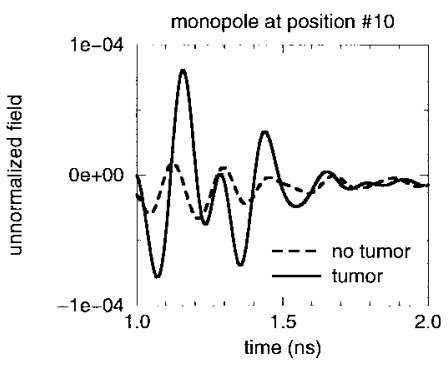

(c)

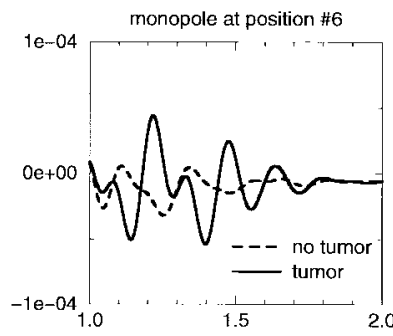

(b)

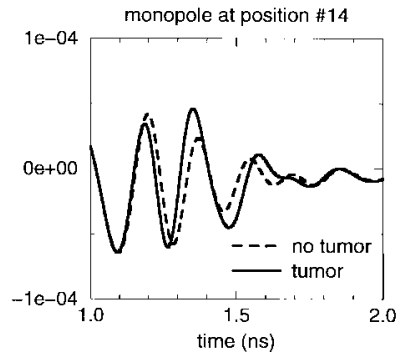

(d)
Fig. 9. FDTD-computed raw data for 4 sample monopole positions of Fig. 8(a) showing the cluttered backscattered responses before time-shifting and summing.

\section{Effect of the Normal Breast Tissue Having Debye Dielectric Dispersion}

In this simulation, the heterogeneous normal breast tissue was assumed to have a Debye dispersion of $\epsilon_{r}$ given by (1) (graphed in Fig. 3). As discussed in Section II, this dispersion reasonably fits the experimental data of Joines et al. [27] and Chaudhary et al. [28]. Using the auxiliary differential equation method [42], the FDTD model incorporated the Debye dispersion separately in each of the $0.5 \mathrm{~cm} \times 0.5$ $\mathrm{cm}$ checkerboard breast-tissue squares shown in Fig. 6(a). Each breast-tissue square was programmed with a unique and randomly selected perturbation (up to $\pm 10 \%$ ) about the nominal $\epsilon_{s}, \epsilon_{\infty}$, and $\sigma$. All electric field components within the given square were updated using the Debye parameters, $\epsilon_{s}, \epsilon_{\infty}$, and $\sigma$, that had been randomly assigned to that square, and each Debye parameter for the square was perturbed by the same percentage relative to the nominal.

Fig. 10 graphs the received power waveform upon coherently summing over the 17 monopoles. There is seen to be no degradation of the $\mathrm{S} / \mathrm{C}$ ratio relative to the corresponding nondispersive modeling results of Fig. 6(b). This is a key result that has favorable implications for our proposed pulsed confocal microwave technology. We believe that the maintenance of the $\mathrm{S} / \mathrm{C}$ ratio in the presence of a realistic Debye breast-tissue dispersion can be explained by the following.

1) The dispersion caused less path loss below the $6-\mathrm{GHz}$ carrier frequency of the microwave signal than was experienced for the nondispersive case of Fig. 6(b). For the assumed symmetric DSB spectrum of the illuminating signal, the total power reaching the tumor for the dispersive case was apparently about the same as for the nondispersive case, but concentrated more in the lower sideband (4-6 GHz).

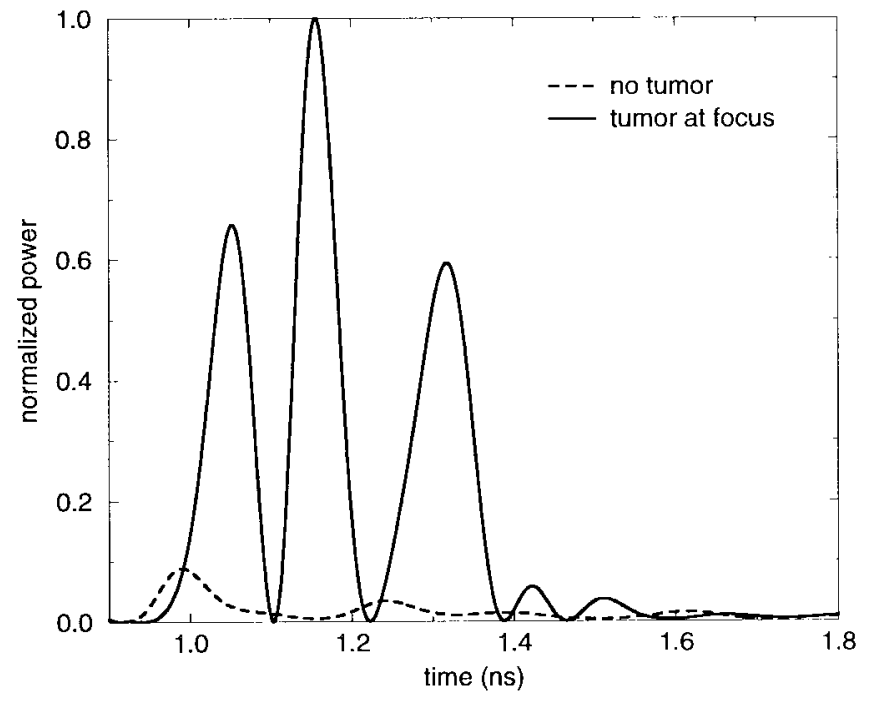

Fig. 10. FDTD-computed time-domain waveforms resulting from time-shifting and summing the backscattered responses for the 17-position coherent-addition antenna array of Fig. 6(a) for a $0.5-\mathrm{cm}$-diameter tumor located at the synthetic focus $3.0 \mathrm{~cm}$ beneath the surface. Here, however, the heterogeneous normal breast tissue is modeled with a Debye dielectric dispersion given by (1).

2) For the realistic Debye parameters modeled, the suppression of the upper sideband $(6-8 \mathrm{GHz})$ of the microwave signal relative to the lower sideband (4-6 GHz) was limited to less than $8 \mathrm{~dB}$ over the $6-\mathrm{cm}$ round-trip path from antenna to tumor to antenna. Sufficient phase coherency of the received backscattered waveform remained after this weak upper-sideband suppression to permit the process of time-shifting and summing the monopole responses to remain effective.

An implication of this result is that the family of noncarrierbased illuminating signals having asymmetrical spectra may not be as robust relative to the breast-tissue dispersion as the simple, symmetrical DSB signal when applying the timeshift and coherent sum strategy for a multiple-element antenna array.

\section{SUMMARY AND CONCLUSIONS}

In this paper, we reported 2-D FDTD modeling of a novel pulsed confocal microwave system for detecting breast cancer. The system exploits breast-tissue physical properties unique to the microwave spectrum, namely, the translucent nature of normal breast tissues (without lesions) and the high dielectric contrast between malignant tumors and the surrounding normal breast tissues. The confocal approach and time-gating permits enhancement of the backscatter detection of malignant tumors while suppressing clutter due to the heterogeneity of the surrounding tissue. Using published data for the dielectric properties of normal and malignant breast tissues, our simulations showed that malignant tumors as small as $2 \mathrm{~mm}$ in diameter can be detected in the presence of the background clutter generated by the heterogeneity. The lateral spatial resolution of the tumor location was found to be about $0.5 \mathrm{~cm}$. Further, the process of time-shifting and coherent summing of the individual backscatter responses of the elements of the 
sensor array, which is fundamental to achieve variable focus in our technology, is shown to be robust relative to: 1) the skin conductivity; 2) the heterogeneity of the healthy-breasttissue $\epsilon_{r}$ and $\sigma$ for random fluctuations up to $\pm 20 \%$ about the mean (more than twice the published values); and c) the Debye dielectric dispersion of the normal breast tissue.

Our initial 3-D FDTD modeling work has analyzed a miniature resistively loaded bow-tie antenna element [43]. We are conducting an ongoing 3-D modeling program to better understand such key system features as wideband bandpass antenna-array design, tumor backscatter dynamic range, effects of tissue clutter and dispersion, and the development of tumorlocation and imaging algorithms. Results from these activities will be reported in later papers. Overall, our 3-D simulation results have been encouraging, and we believe that the pulsed confocal microwave tumor-detection technology has good promise for eventual transition to preclinical trials.

\section{ACKNOWLEDGMENT}

The authors would like to thank Prof. A. Sahakian of Northwestern University, Prof. M. Griem of the University of Chicago, and Dr. J. Aarsvold of the Atlanta VA Medical Center for their guidance and consulting, as well as the technical support of M. Popovic of Northwestern University. They would also like to thank Cray Research, Inc., for providing computing resources.

\section{REFERENCES}

[1] J. E. Bridges, "Noninvasive system for breast cancer detection," U.S. Patent 5704 355, Jan. 6, 1998.

[2] J. W. Lichman, "Confocal microscopy," Scientif. American, vol. 171, pp. 40-45, 1994.

[3] J. O. Enk, G. T. Dubiel, and J. E. Bridges, "Millimeter-wave FM radar weapons detection system," Final Report, FAA Contract DTFA03-87C-00056, July 1992.

[4] J. E. Bridges, "Confocal, synthetic-impulse, millimeter wave system for imaging concealed dielectric and metallic objects," in Proc. North American Radio Science Meeting, Montreal, Canada, July 1997, paper A58.4.

[5] D. B. Kopans, "Early breast cancer detection using techniques other than mammography," Amer. J. Roentgenol., vol. 143, pp. 465-468, Sept. 1984.

[6] S. H. Heywang-Köbrunner, "Nonmammographic breast imaging techniques," Current Opinion in Radiol., vol. 4, pp. 146-154, Oct. 1992.

[7] M. Säbel and H. Aichinger, "Recent developments in breast imaging," Phys. Med. Biol., vol. 41, pp. 315-368, Mar. 1996

[8] A. H. Barrett and P. C. Myers, "Basic principles and applications of microwave thermography," in Medical Applications of Microwave Imaging, L. E. Larsen and J. H. Jacobi, Eds. Piscataway, NJ: IEEE Press, 1986, pp. 41-46.

[9] L. E. Larsen and J. H. Jacobi, Eds., Medical Applications of Microwave Imaging. Piscataway, NJ: IEEE Press, 1986.

[10] R. E. Sepponen, "Medical diagnostic microwave scanning apparatus," U.S. Patent 4641659 , Feb. 10, 1987.

[11] A. W. Preece, H. Johnson, F. L. Green, and M. P. Robinson, "Dielectric imaging for localization and detection of breast tumors," in Proc. IEEE MTT Int. Microwave Symp. Dig., 1993, pp. 1145-1146.

[12] B. D. Sollish, E. H. Frie, E. Hammerman, S. B. Lang, and M. Moshitsky, "Microprocessor-assisted screening technique," Israel J. Med. Sci., vol. 17, pp. 859-864, 1981

[13] W. Guo and T. Guo, "Quantitative dielectric imaging system," U.S. Patent 5363 050, Nov. 8, 1994.

[14] J. D. Young and L. Peters, Jr., "Examination of video pulse radar systems as potential biological exploratory tools," in Medical Applications of Microwave Imaging, L. E. Larsen and J. H. Jacobi, Eds. Piscataway, NJ: IEEE Press, 1986, pp. 82-105.
[15] R. W. Cribbs, "New concepts for the location of underground plastic natural gas pipes," Gas Research Institute, Chicago, IL, Final Report, Contract 5094-260-2894, Dec. 1995

[16] T. P. Montoya and G. S. Smith, "Mine detection using vee dipoles in a short-pulse ground penetrating radar," in Proc. North American Radio Science Meeting, Montreal, Canada, July 1997, paper A58.1.

[17] H. Brunzell, "Inpulse radar for detection and classification of buried land mines," in Proc. North American Radio Science Meeting, Montreal, Canada, July 1997, paper A58.2.

[18] F. C. Chen and W. C. Chew, "Ultra-wideband imaging radar," in Proc. North American Radio Science Meeting, Montreal, Canada, July 1997, paper A58.6.

[19] J. F. Aurand, "Ultra-wideband transient backscatter measurements through concrete walls," in Proc. North American Radio Science Meeting, Montreal, Canada, July 1997, paper A58.7.

[20] G. S. Wallinga, E. J. Rothwell, K. M. Chen, and D. P. Nyquist, "Application of cepstral analysis to radar target discrimination," in Proc. North American Radio Science Meeting, Montreal, Canada, July 1997, paper A58.8.

[21] W. T. Clark, R. L. Hutchings, J. S. Schoenberg, and J. S. Tyo, "Characterization of off-boresight radiation from an impulse excited TEM horn," in Proc. North American Radio Science Meeting, Montreal, Canada, July 1997, paper A58.10.

[22] P. Kearey and M. Brooks, "Seismic reflection surveying," in Geoscience Texts, Vol. 4: An Introduction to Geophysical Exploration. Boston, MA: Blackwell, 1984, ch. 4.

[23] C. Gabriel, "A compilation of the dielectric properties of body tissues at RF and microwave frequencies," Radiofrequency Radiation Division, Brooks AFB, San Antonio, TX, Contract AL/OE-TR-1996-0037, 1996.

[24] C. Gabriel, S. Gabriel, and E. Corthout, "The dielectric properties of biological tissues: I. Literature survey," Phys. Med., Biol., vol. 41, no. 11, pp. 2231-2249, Nov. 1996.

[25] S. Gabriel, R. W. Lau, and C. Gabriel, "The dielectric properties of biological tissues: II. Measurements on the frequency range $10 \mathrm{~Hz}$ to 20 GHz," Phys. Med., Biol., vol. 41, no. 11, pp. 2251-2269, Nov. 1996.

[26] _ _ "The dielectric properties of biological tissues: III. Parametric models for the dielectric spectrum of tissues," Phys. Med., Biol., vol. 41, no. 11, pp. 2271-2293, Nov. 1996.

[27] W. T. Joines, Y. Z. Dhenxing, and R. L. Jirtle, "The measured electrical properties of normal and malignant human tissues from 50 to $900 \mathrm{MHz}$," Med. Phys., vol. 21, pp. 547-550, Apr. 1994.

[28] S. S. Chaudhary, R. K. Mishra, A. Swarup, and J. M. Thomas, "Dielectric properties of normal and malignant human breast tissues at radiowave and microwave frequencies," Indian J. Biochem., Biophys., vol. 21, pp. 76-79, Feb. 1984

[29] K. R. Foster and J. L. Schepps, "Dielectric properties of tumor and normal tissue at radio through microwave frequency," J. Microwave Power, vol. 16, pp. 107-119, 1981.

[30] J. A. Rogers, R. J. Shepard, E. H. Grant, N. M. Bleehen, and D. J. Honess, "The dielectric properties of normal and tumor mouse tissue between $50 \mathrm{MHz}$ and $10 \mathrm{GHz}$," Br. J. Radiol., vol. 56, pp. 335-338, 1983.

[31] R. Peloso, D. Tuma, and R. K. Jain, "Dielectric properties of solid tumors during normothermia and hyperthermia," IEEE Trans. Biomed. Eng., vol. BME-31, pp. 725-728, 1984.

[32] A. S. Swarup, S. S. Stuchly, and A. Surowiec, "Dielectric properties of mouse MCA1 fibrosarcoma at different stages of development," Bioelectromagn., vol. 12, pp. 1-8, 1991.

[33] A. J. Surowiec, S. S. Stuchly, J. R. Barr, and A. Swarup, "Dielectric properties of breast carcinoma and the surrounding tissues," IEEE Trans. Biomed. Eng., vol. 35, pp. 257-263, Apr. 1988

[34] K. R. Foster and H. P. Schwan, "Dielectric properties of tissues and biological materials: a critical review," Crit. Rev. Biomed. Eng., vol. 17, pp. $25-104,1989$

[35] C. C. Johnson and A. W. Guy, "Nonionizing electromagnetic wave effects in biological materials and systems," Proc. IEEE, vol. 60, pp. 692-718, 1972.

[36] H. Gray, Anatomy of the Human Body, C. M. Goss, Ed. Philadelphia, PA: Lea and Febiger, 1949.

[37] X. Wu, G. T. Barnes, and D. M. Tucker, "Spectral dependence of glandular tissue dose in screen-film mammography," Radiol., vol. 179, pp. 143-148, Apr. 1991

[38] M. Rosenstein, L. W. Andersen, and G. Warner, "Handbook of glandular tissue doses in mammography," U.S. Government Printing Office, Washington, DC, HHS (FDA) Publication 858239, 1985.

[39] W. H. Parsons, Cancer of the Breast. Springfield, IL: Thomas, 1959.

[40] S. C. Hagness, A. Taflove, and J. E. Bridges, "FDTD analysis of a pulsed microwave confocal system for breast cancer detection," in Proc. Int. 
Conf. IEEE Engineering in Medicine and Biology Society, Chicago, IL, Oct. 1997 , pp. $2506-2508$.

[41] A. Taflove, Computational Electrodynamics: The Finite-Difference Time-Domain Method. Boston, MA: Artech House, 1995.

[42] R. M. Joseph, S. C. Hagness, and A. Taflove, "Direct time integration of Maxwell's equations in linear dispersive media with absorption for scattering and propagation of femtosecond electromagnetic pulses," Optics Lett., vol. 16, no. 18, pp. 1412-1414, 1991.

[43] S. C. Hagness, A. Taflove, and J. E. Bridges, "Wideband ultralow reverberation antenna for biological sensing," Electron. Lett., vol. 33, no. 19 , pp. 1594-1595, 1997.

Susan C. Hagness (S'91-M'98), for a photograph and biography, see p. 1076 of the August 1998 issue of this Transactions.

Allen Taflove (M'75-SM'84-F'90), for a photograph and biography, see $\mathrm{p}$. 1076 of the August 1998 issue of this TRANSACTIONS.

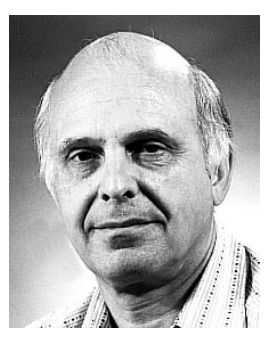

Jack E. Bridges (A'50-M'55-SM'56-F'74-LF'91) was born in Denver, CO, on January 6, 1925. He received the B.S. and M.S. degrees in electrical engineering in 1945 and 1947, respectively, from the University of Colorado, Boulder.

Prior to 1961, he was employed by Andrew Corp., Zenith Radio, and Warwick Manufacturing. From 1961-1992, he was with IIT Research Institute, where he attained the rank of Senior Science Advisor and initiated new interdisciplinary technologies, including an electrical method to stimulate flow rates from oil wells and an RF low-temperature method to sterilize medical wastes. In 1993, he founded Interstitial, Inc., to develop novel technologies in the energy and biomedical fields. He is an author of more than 100 journal articles or presentations, and he currently holds 64 U.S. patents.

Mr. Bridges was the recipient of the 1983 Distinguished Engineering Alumnus Award from the University of Colorado; the 1981 Power Engineering Society Best Paper Award for "Biological Effects of ELF Electric Fields"; and the 1956 Browder J. Thompson Best Paper Award for "Detection of Television Signals in Thermal Noise." He is a member of Eta Kappa Nu, Tau Beta Pi, Sigma Xi, the Bioelectromagnetics Society, and the American Association of Physicists in Medicine. 